\title{
Analgesia Regional Prolongada com Catéteres Periféricos. Relato de Casos *
}

\section{Prolonged Regional Analgesia with Peripheral Catheters. Case Reports}

\author{
Karl Otto Geier ${ }^{1}$
}

\section{RESUMO}

Geier KO - Analgesia Regional Prolongada com Catéteres Periféricos. Relato de Casos

Justificativa e Objetivos - Síndromes de Dor de Manutenção Simpática, Tromboangeíte Obliterante, Esclerodermia Sistêmica Progressiva (doença autoimune) e analgesia pós-operatória respondem muito bem aos anestésicos locais quando em uso prolongado. O objetivo dos casos relatados visa divulgar o uso de catéteres curtos (catéteres venosos) ou segmento de cateter peridural nas situações acima, na falta de material apropriado (Contiplex ${ }^{\circledR}$ e similares) associados ao estimulador de nervo periférico.

Relato dos Casos - São relatados casos de Síndrome de Dor Regional Complexa 1 e 2, úlcera de perna por tromboangeíte obliterante para preparação a enxertia de pele, esclerodermia sistêmica progressiva com comprometimento microcirculatório distal do pé, em que foram colocados catéteres venosos contíguos aos nervos periféricos dos respectivos territórios, visando o controle contínuo da dor através de injeções de anestésicos locais.

Conclusões - Baseados nos princípios que norteiam a boa prática de anestesia regional e nos resultados obtidos, os casos apresentados mostraram que os catéteres usados podem substituir aqueles especialmente designados para as mesmas finalidades.

UNITERMOS - DOR: crônica; TÉCNICAS ANESTÉSICAS, Regional: continua

\section{INTRODUÇÃO}

$\mathrm{O}$ bter analgesia regional prolongada em áreas periféricas sem comprometer sistemicamente outros parâmetros fisiológicos que resultam em repercussões hemodinâmicas, vasculares ou respiratórias sempre foi um objetivo dos anestesiologistas. Situações clínicas como: analgesia pós-operatória, troca de curativos dolorosos, reintervenções cirúrgicas seriadas, fisioterapia indolor e sedação da dor aguda e/ou crônica são situações comuns no ambiente

\footnotetext{
* Recebido do (Received from) Serviço de Dor do Hospital São Lucas da Pontifícia Universidade Católica do Rio Grande do Sul, RS

1. Médico Colaborador da Clindor/PUCRS
}

Apresentado (Submitted) em 16 de fevereiro de 2001

Aceito (Accepted) para publicação em 23 de julho de 2001

Correspondência para (Mail to):

Dr. Karl Otto Geier

Rua Cel. Camisão, 172

90540-030 Porto Alegre, RS

E-mail: carlotto@portoweb.com.br

(c) Sociedade Brasileira de Anestesiologia, 2002

\section{SUMMARY}

Geier KO - Prolonged Regional Analgesia with Peripheral Catheters. Case Reports

Background and Objectives - Sympathetic Maintenance Pain Syndrome, Obliterating Thromboangiitis, Progressive Systemic Scleroderma (auto-immune disease) and postoperative analgesia are good responders to prolonged local anesthetics. These reports aimed at spreading the use of short catheters (venous catheters) or epidural catheter segments associated to peripheral nerve stimulator in the above mentioned situations and when adequate material (Contplex ${ }^{\circledR}$ and similar equipment) are not available.

Case Reports - Cases of Complex Regional Pain 1 and 2, leg ulcer by obliterating thromboangiitis in preparation to skin graft, and progressive systemic scleroderma with distal foot microcirculatory involvement are reported in which venous catheters were inserted close to the peripheral nerves of the respective territories aiming at continuously controlling pain through local anesthetic injections.

Conclusions - Based on regional anesthesia best practices and on the results obtained, all cases reported have shown that investigated catheters may replace those specially designed for the same purposes.

KEY WORDS - ANESTHETIC TECHNIQUES, Regional:continuous; PAIN: cronic

hospitalar e em clínicas de dor. Desafortunadamente, face à escassez ou mesmo à inexistência de material compatível e de técnicas anestesiológicas regionais específicas durante a última década, a execução da maioria desses procedimentos nas situações citadas era inviável. A analgesia regional alternativa, promovida por punções repetitivas, era desconfortante e dolorosa para o paciente e laboriosa para o anestesiologista. Anestésicos locais de longa duração, administrados em bolus, tinham ação limitada e a analgesia/anestesia prolongada exigia a utilização de catéteres periféricos. Os requisitos básicos para a prática e o bom desempenho da anestesia regional resumiam-se, portanto, aos conhecimentos anatômicos, à fisiologia da fibra nervosa, ao armamentário, à experiência e ao domínio individual do anestesiologista com as diversas técnicas anestésicas regionais. Todavia, mesmo na ausência de material mais adequado, é possível realizar bloqueios e técnicas contínuas. O objetivo desses relatos é mostrar que o uso de catéteres venosos ou segmento de cateter peridural colocados contíguos a nervos periféricos ou plexos nervosos tem finalidade profilática (dor fantasma), diagnóstica (dor somática da dor simpática), terapêutica (analgesia pós-operatória) e prog- 
nóstica (evolução das síndromes dolorosas por manutenção simpática).

\section{RELATO DOS CASOS}

Caso 1 - Paciente com 28 anos, operadora de linha de montagem de uma indústria de calçados, ao acionar a máquina em que trabalhava, teve seu polegar totalmente luxado na região de articulação metacarpofalangeana. Embora reduzido incruentamente instantes após, a paciente continuava referindo dor contínua do tipo "ardência" e incapacidade física durante os dias subseqüentes. Foi operada visando "reforço articular". No pós-operatório, o quadro se agravou quando foi encaminhada ao Serviço de Dor. Na ocasião, apresentava a mão fria, sudorética, cianótica e com manifestações de alodínia e hiperalgia, diagnosticando-se Síndrome de Dor Regional Complexa do tipo 1. No cotovelo, foi colocado um cateter venoso $18 \mathrm{G}$ contíguo aos dermátomos e angiótomos correspondentes aos territórios dos nervos radial e músculocutâneo, objetivando sedação da atividade simpática com bupivacaína a $0,25 \%, 8 \mathrm{ml}$, até quatro vezes por dia. O bloqueio periférico do nervo radial foi inicialmente realizado pela punção com agulha $25 \times 7$, dois centímetros acima da prega cutânea articular, face lateral ao tendão do bíceps. Com a agulha nessa posição, injetou-se uma dose teste analgésica, observando analgesia em poucos minutos. Aseguir, substituiu-se a agulha por um cateter venoso reproduzindo, cuidadosamente, a punção anterior, até detectar perda de resistência entre ao planos musculares (aproximadamente $2 \mathrm{~cm}$ da pele). $O$ cateter foi introduzido e fixado. Estudo radiológico comprovou o correto posicionamento do cateter (Figura 1). Após cada injeção anestésica, observaram-se vasodilatação, rubor e analgesia dos territórios envolvidos. O tratamento foi completado com bloqueios intravenosos regionais de anestésico local e guanetidina. Adicionalmente, sob o efeito do bloqueio regional via cateter, iniciou-se fisioterapia indolor. Ao final de duas semanas, o quadro regrediu sensivelmente.

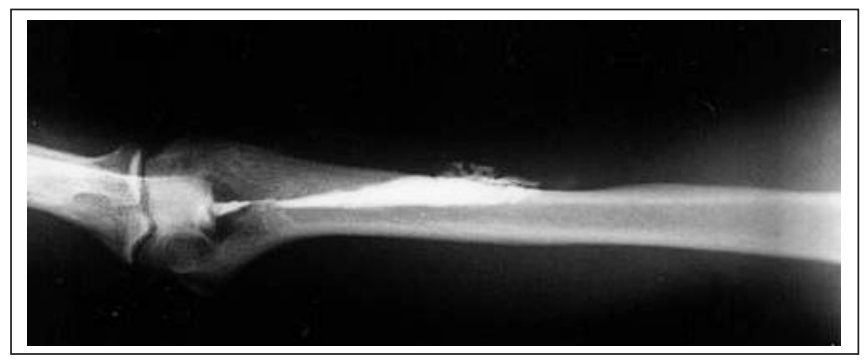

Figura 1 - Radiografia de Perfil Identificando o Cateter Venoso e Contraste na Altura dos Nervos Radial e Músculo-cutâneo em Paciente com Síndrome de Dor Regional Complexa 1

Caso 2 - Paciente com 45 anos, masculino, acidentado de trânsito, teve importante ruptura parcial do manguito rotador do ombro direito. Foi operado, necessitando de fisioterapia precoce no pós-operatório imediato. Impossibilitado de realizar movimentos ativos e passivos da articulação escápulo-umeral face à dor, foi conduzido ao Serviço de Dor do Hos- pital. Após devidamente avaliado, decidiu-se colocar um cateter venoso $18 \mathrm{G}$ na fossa supra-espinhal da escápula, contíguo ao nervo supraescapular, visando fisioterapia indolor passiva e ativa do ombro. Bupivacaína a $0,25 \%$ com adrenalina a 1:200.000, $5 \mathrm{ml}, 20$ minutos antes da fisioterapia permitiu a realização de movimentos ativos indolores por três dias, quand o o cateter foi deslocado ao exterior. O tratamento continuou com antiinflamatórios não esteróides e analgésicos.

Caso 3 - Paciente com 17 anos, feminina, atingida por uma bala perdida que transfixou o músculo grande peitoral e braço esquerdo. Os batimentos arteriais periféricos tornaram-se comprometidos. Foi submetida à revascularização de urgência da artéria subclávia com autoenxerto venoso. Menos de 24 horas de pós-operatório apareceu dor contínua, em queimadura, hiperalgia ${ }^{8}$ e alodínia ${ }^{8}$ na mão e antebraço. Inicialmente foi tratada com analgésicos e antiinflamatórios não esteróides (AINS), porém, sem sucesso. Quarenta e oito horas de pós-operatório, foi contatado o Serviço de Dor que diagnosticou tratar-se de Síndrome Dor Regional Complexa 2 do membro superior esquerdo. Instituído tratamento por três dias com uma série de bloqueios intermitentes do simpático cérvico-torácico esquerdo através de um segmento de cateter peridural, introduzido através de um cateter venoso $18 \mathrm{G}$ previamente colocado em C6-C7 na altura do gânglio estrelado. Embora o alívio da dor não tenha sido de longa duração, a analgesia foi efetiva, notadamente em relação à alodínia. Quando o cateter deslocou-se ao exterior, no final do terceiro dia, os procedimentos foram substituídos por bloqueios intermitentes do plexo braquial, durante seis dias, através de um cateter venoso introduzido na fenda interescalênica. Bupivacaína a $0,125 \%$, com adrenalina a $1: 400.000,8 \mathrm{ml}$ e bupivacaína a 0,25\% com adrenalina a $1: 200.000,30 \mathrm{ml}$, ambas de $8 / 8$ horas, foram as soluções anestésicas utilizadas, respectivamente, para sedação da atividade simpática (gânglio estrelado) e analgesia da dor somática do membro superior esquerdo (plexo braquial) (Figura 3). Sob o novo tipo de analgesia (plexo braquial), iniciou-se fisioterapia precoce. Nove dias após, a paciente teve alta hospitalar com acentuada melhora do quadro álgico. Caso 4 - Paciente com 54 anos, portador de enorme úlcera dolorosa de perna de etiologia arterioesclerótica, situada no terço inferior da perna, nos territórios supridos pelos nervos periféricos do safeno interno e fibular $D$, tinha sido submetido a "by-pass" fêmuro-poplíteo. No pós-operatório, infecção cirúrgica comprometeu a revascularização, necessitando uma derivação maior, ilíaco poplítea, porém ainda sem bons resultados. Dois enxertos livres de pele sobre a úlcera resultaram cerca de $80 \%$ de rejeição. O membro inferior direito persistia pálido, frio, doloroso e edematoso. Sem perspectiva de resolução de seu quadro clínico, era candidato à amputação do membro. Contatado o Serviço de Dor mais pela queixa de sua dor, foi submetido à colocação de catéteres venosos $18 \mathrm{G}$ contíguos aos referidos nervos. Para o nervo fibular, o cateter foi colocado na altura da cabeça da fíbula e para o nervo safeno interno, a $8 \mathrm{~cm}$ acima da linha articular fêmorotibial pela técnica transartorial. Após uma dose de lidocaína a 1,5\%, $8 \mathrm{ml} \mathrm{em}$ cada cateter, o quadro doloroso evoluiu para 
boa analgesia. Manteve-se uma perfusão contínua com baixa concentração de lidocaína sob bombas de infusão em cada cateter. Seis horas depois, o resultado foi surpreendente. A perna direita apresentava-se com analgesia, quente, ruborizada e a úlcera menos isquêmica. Aconduta permaneceu durante 24 horas quando nova enxertia foi realizada. Os catéteres proporcionaram anestesia cirúrgica e analgesia pós-operatória. Teve alta hospitalar no dia seguinte, sendo seus familiares instruídos a continuar com a mesma analgesia via catéteres, com doses fracionadas. Retornou no sétimo dia de pós-operatório para revisão. De acordo com o cirurgião, o enxerto de pele sobre a úlcera demonstrava $70 \%$ de integração.

Caso 5 - Paciente com 7 anos, masculino, foi atingido acidentalmente por um disparo de espingarda, no flanco direito, de cima para baixo em posição médio-lateral, com penetração abdominal baixa e saída na altura da face lateral da coxa, com destruição completa da cabeça do fêmur. Foi submetido à laparotomia e posteriormente à tração transesquelética do membro inferior direito com $5 \mathrm{~kg}$ de peso. Ador da incisão abdominal diminuiu após o terceiro dia. Continuava, porém, com dor intensa na "articulação coxo-femoral", que em seguida expôs um trajeto fistuloso purulento pelo orifício de saída do projétil. Estava em uso medicação opióide. No décimo dia apresentava-se assintomático em relação à laparotomia, contudo a dor do fêmur era insuportável. Contatado o Serviço de Dor que diagnosticou dor por excesso de nocicepção (drenagem purulenta). Foi realizado bloqueio diagnóstico com lidocaína a 1,5\%, $5 \mathrm{ml}$ no nervo femoral. Após latência de quatro minutos, a dor foi diminuindo até o completo alívio no décimo minuto. Substituiu-se a agulha $25 \times 7$ que permaneceu no local, por um cateter venoso $20 \mathrm{G}$, reproduzindo a mesma punção com o objetivo de anestesiar todo o território somático suprido pelo nervo femoral. Bloqueios intermitentes com bupivacaína a 0,25\% com adrenalina a 1:200.000, 8 $\mathrm{ml}$, cada 6 horas nas primeiras 24 horas e depois sob infusão contínua mediante bomba de infusão, resultaram surpreendente analgesia, inclusive durante as trocas de curativos. Infelizmente, no segundo dia, o cateter foi expulso do local, sendo necessário substituí-lo por outro. Um cateter peridural foi colocado no mesmo espaço através de outro cateter venoso que serviu como guia à semelhança do Caso 3 . Com o cateter periférico foi possível anestesia cirúrgica para os desbridamentos da fístula e analgesia pós-operatória desses procedimentos. No décimo quinto dia, ainda sob tração esquelética, o paciente apresentava-se melhor e a fístula antes purulenta estava quase cicatrizada.

Caso 6 - Paciente com 78 anos, portadora de Lupus Eritematoso Sistêmico e Esclerodermia associados, ambas doenças autoimunes, cujo passado mórbido revelou: 1) manifestações de Síndrome de Raynaud em ambas as mãos, efetivamente tratadas com uma série de bloqueios anestésicos sobre a cadeia simpático cérvico-torácica (gânglio estrelado), 2) várias cirurgias vasculares no membro inferior direito pelo mesmo motivo. Recentemente, face a distúrbios circulatórios progressivos peculiares às doenças autoimunes, teve seu membro inferior direito amputado acima do joelhoe,
3) amputação do segundo dedo do pé esquerdo devido a uma úlcera com necrose do hálux por falência circulatória distal. Com dor isquêmica no terceiro dedo do mesmo pé que evoluía também para necrose, foi encaminhada ao Serviço de Dor. Ao exame, a dor era insuportável. O tratamento foi iniciado com uma série de bloqueios de Bier com anestésico local, guanetidina e um dilatador da microcirculação. Em continuação, um cateter venoso $20 \mathrm{G}$ foi colocado contíguo ao nervo tibial posterior, no maléolo interno, visando analgesia e bloqueio do angiótomo correspondente. Um bloqueio prova com bupivacaína a $0,25 \%, 6 \mathrm{ml}$, resultou abolição de sua dor. A analgesia se manteve com infusões a cada 6 horas com a mesma solução e após, sob solicitação da paciente. Delimitado o processo necrótico no terceiro dedo, não foi necessária a amputação do pé como inicialmente programado e, sim, apenas do dedo. Com três dias de uso, o cateter foi expulso do local, sendo também substituído por outro cateter venoso. O segundo cateter permaneceu in situ durante nove dias, e foi útil na analgesia preemptiva, na cirurgia propriamente dita (amputação do terceiro dedo), na analgesia pós-operatória e de toda dor isquêmica residual.

\section{DISCUSSÃO}

Dentre a classificação de Síndromes de Dor Regional Complexas ${ }^{1,6}$, a Síndrome Dor Regional Complexa 1, antes denominada de Distrofia Simpático Reflexa, corresponde a uma desordem neurológica originada por um evento nociceptivo traumático ou cirúrgico, com repercussões clinico patológicas manifestadas por dor e alterações sensoriais desproporcionadas em intensidade, distribuição e duração 2,7. O evento nociceptivo etiológico da paciente do Caso 1 foi o trauma. Adicionalmente, constatam-se nessa síndrome disfunções motoras, autonômicas, tróficas e psicológicas que culminam na incapacidade física regional. A dor é intensa sob forma de ardência extremamente desconfortável. Ocorre alodínia ${ }^{3,8}$, hiperestesia ${ }^{3,8}$, edema, distrofia pelo repouso da área acometida, descontrole vasomotor e sudomotor (hiperidrose ou anidrose) da região afetada pela hiperatividade autonômica simpática, fenômenos que a paciente apresentava. O quadro finalmente evolui para rigidez ou "congelamento" articular com osteoporose avançada, revelada por exame radiológico. Acomete mais as extremidades, mãos e dedos; ainda ombros, articulação coxofemoral e, eventualmente, região peitoral pós-mastectomia. As Síndromes de Dor Regional Complexas agravam-se após eventos nociceptivos reincidentes nas regiões em atividade e sob terapêutica insuficiente ou ainda virgens de tratamento. O diagnóstico é elaborado pela história clínica e exame físico, sendo relevante um evento traumático ou nociceptivo. No Caso 1, a alodínia surgia após um simples sopro (alodínia aeólica) sobre as áreas comprometidas (dermátomos). O tratamento consistiu na analgesia troncular e venosa regional ${ }^{4,5,9,10}$ respectivamente, com anestésicos locais e anestésico local/guanetidina, sedação da hiperatividade simpática e fisioterapia indolor precoce (passiva e ativa) sob analgesia. Foi o que realizamos na paciente. História clínica e exaVol. 52, Nº 1, Janeiro - Fevereiro, 2002 
me físico associados a bloqueios anestésicos regionais periféricos conduzem, com grande precisão, ao diagnóstico ${ }^{5,10}$. A repetição dos mesmos bloqueios via cateter (Figura 1) influenciam na terapêutica e no prognóstico da entidade.

O manguito rotator recebe importante inervação do nervo supraescapular (C4-C6). É um tendão conjunto composto pelos músculos rotadores supra e infraespinhoso, pequeno redondo e subescapular, constituindo a "coifa" da articulação escápulo-umeral ${ }^{6,11}$. Lesões do tipo estiramento, traumas com lesão completa ou incompleta que resultam em inflamação, fibrose ou degeneração cartilaginosa da articulação gleno-umeral são importantes etiologias que afetam o manguito rotator. O trauma envolvendo o paciente do Caso 2 é a principal causa de hipovascularização devido à compressão constante, envolvendo a grande tuberosidade e as porções anterior e inferior do acrômio sobre a inserção do músculo supraespinoso comprometendo sua nutrição. Outra causa importante é o impacto subacromial primário com degeneração do manguito rotator, cuja área de conflito situa-se na inserção do músculo supraespinoso e a cabeça longa do bíceps. O atrito continuado desses músculos contra o arco acromial resulta comprometimento circulatório e degeneração do manguito rotator ${ }^{7,12}$. Quando se constata a ruptura, a indicação geralmente é cirúrgica, seguida precocemente pela fisioterapia. A fisioterapia é importante, pois com a movimentação precoce da articulação escápulo-umeral, previne-se o ombro "congelado". A analgesia do manguito rotator pode ser obtida pelo bloqueio do plexo braquial por via interescalênica, do próprio nervo supraescapular por via anterior $^{8,13}$ ou posterior $^{9,14}$ ou via intra-articular ${ }^{10,15}$. A primeira opção apresenta como inconveniente, dependendo da massa e da concentração do anestésico local, um leve a moderado bloqueio motor de todo MS, resultando na impossibilidade de movimentação ativa ou na analgesia insuficiente durante a fisioterapia. Como segunda alternativa efetiva, temos o bloqueio do nervo supraescapular. Em nosso Hospital, o manuseio da dor proveniente de fraturas isoladas ou não da escápula é feito com bloqueio contínuo do nervo supraescapular com o paciente sentado embora o decúbito dorsal tenha sido descrito para a mesma doença ${ }^{11,16}$. Os resultados satisfatórios do bloqueio contínuo do nervo supraescapular em outras situações como analgesia pós-operatória ${ }^{10,15}$ e dor oncológica do ombro ${ }^{12,17}$ ampliaram as indicações deste bloqueio.

A abordagem no paciente foi posterior, com uma agulha comum tamanho $25 \times 7$, até obter parestesias na fossa supraespinhal. Essa técnica foi utilizada em outras doenças ${ }^{13,18}$, porém com posicionamento modificado ${ }^{14,19}$. Com a agulha no local, procedeu-se ao teste terapêutico com bupivacaína a $0,25 \%, 3 \mathrm{ml}$. Confirmada analgesia, retiramos a agulha e reproduzimos a punção com um cateter venoso 20G, procurando introduzi-lo até contatar a escápula. Realizamos os primeiros bloqueios com os pacientes sentados, e o acesso à fossa supraespinhal era vertical (Figura 2); porém, atualmente o fazemos horizontalmente. Com o cateter no local, o segmento externo é fixado com fita adesiva e acolchoado com gazes ao seu redor. Doses analgésicas de bupivacaína a $0,25 \%, 5 \mathrm{ml}$ cada 6 horas, proporcionaram a recuperação do paciente.

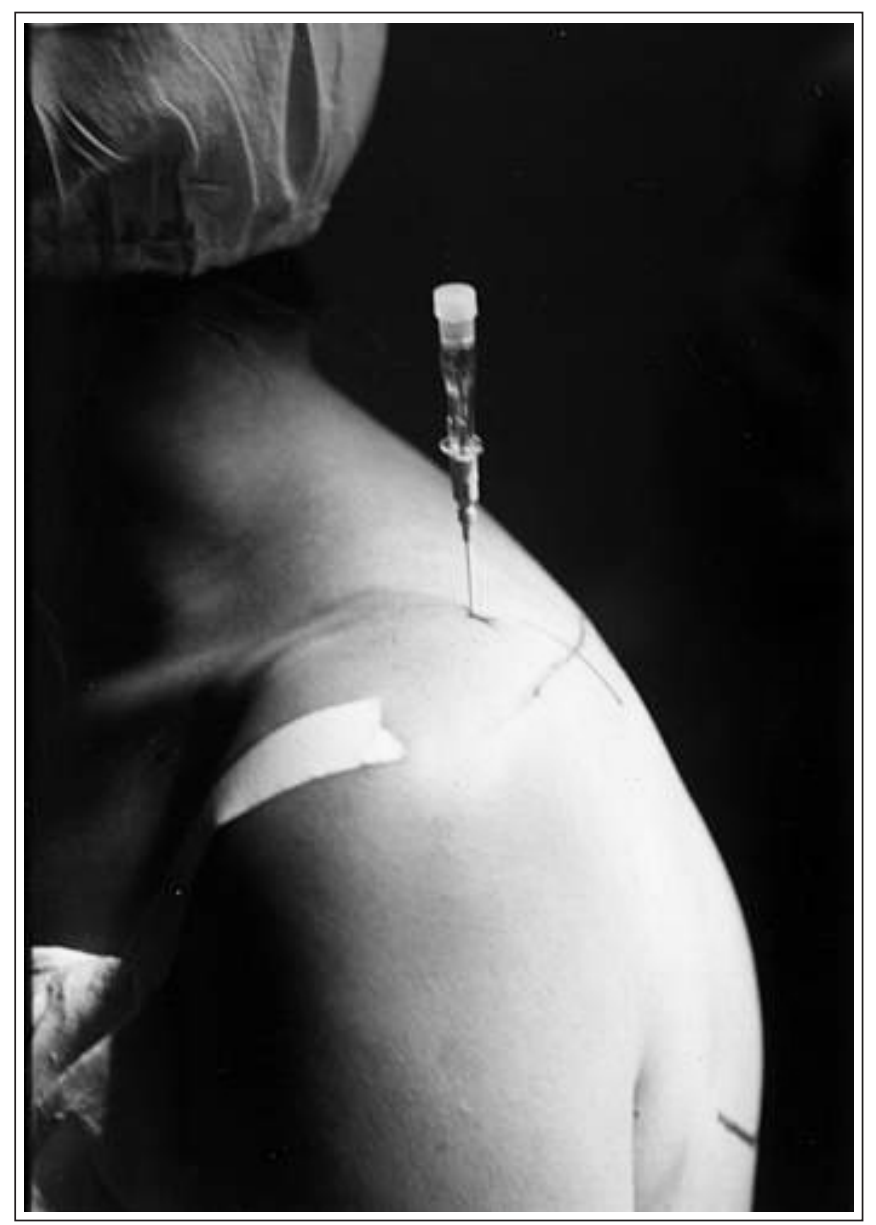

Figura 2 - Cateter Venoso no Espaço Supraespinoso da Escápula Esquerda

Devidamente orientado, paciente e fisiatra realizaram, no consultório e a domicilio, fisioterapia indolor sob bupivacaína durante três dias, enquanto o cateter permaneceu no local. No sétimo dia, o paciente estava praticamente assintomático.

Como no Caso 3, as Síndromes de Dor Regional Complexas abrangem tambéma Síndrome de Dor Regional Complexa 2, anteriormente denominada de causalgia ${ }^{1,6}$, que apresenta como substrato um evento traumático ou cirúrgico porém, acrescido de lesão neural. As manifestações álgicas mais importantes são as disestesias ${ }^{3,8}$ (dores agudas em "fisgadas" ao longo do tecido neural lesado) e a alodínia. SemeIhantemente à Síndrome de Dor Regional Complexa 1, o diagnostico se faz pela história clínica e pelo exame físico. Como queríamos distinguir o tipo e a intensidade dolorosa (dor somática da dor autonômica simpática) ${ }^{15,20}$, iniciamos com bloqueios repetitivos do gânglio estrelado, via cateter, em lugar de sucessivas punções. A seguir, um segmento de cateter peridural foi introduzido via cateter venoso previa- 
mente posicionado e que serviu de guia ao primeiro. A retirada do cateter venoso foi semelhante à técnica de cateterização do espaço peridural. Prolongada sedação da atividade simpática do gânglio estrelado foi possível em ocasiões anteriores ${ }^{16,17,21,22}$. Mediante baixas concentrações de anestésico local, de forma intermitente ou contínua sob regime de Analgesia Controlada pelo Paciente ${ }^{17,22}$, dores de manutenção simpática tornam-se mais controladas. Todavia, a curta permanência dos catéteres in situ, provavelmente pela região anatômica desfavorável, constante movimentação cervical e pela fixação do cateter com material adesivo inviabilizam um tratamento prolongado. Por esse motivo, continuamos o tratamento pela via interescalênica (Figura 3), mediante um cateter venoso $18 \mathrm{G}$ colocado diferentemente da abordagem (sentido da agulha) e técnica (perda de resistência) preconizada por Winnie ${ }^{18,23}$. Esse procedimento com essas características de acesso e técnica é rotineiro em nosso Hospital. A paciente teve alta hospitalar no nono dia com prosseguimento terapêutico fisiátrico em regime ambulatorial.

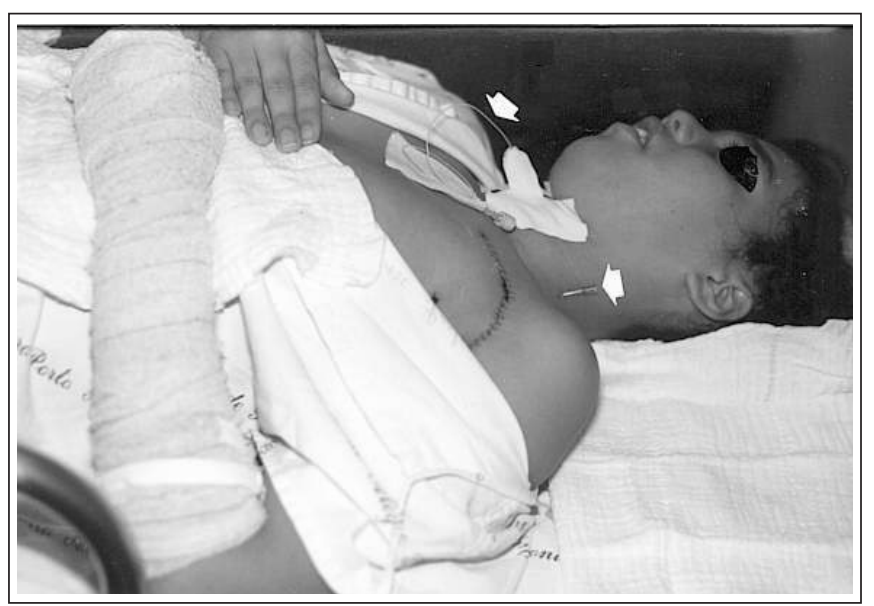

Figura 3 - Paciente com Síndrome de Dor Regional Complexa 2 por arma de fogo que transfixou o grande peitoral com lesão vascular subclávia e plexo braquial. Bloqueio intermitente do gânglio estrelado via segmento de cateter peridural (seta) foi eficiente enquanto esteve in situ. A terapêutica prosseguiu via cateter venoso interescalênico (seta)

Arteriosclerose obliterante é a doença arterial mais comum da aorta abdominal inferior, da artéria ilíaca e de seus ramos nos membros inferiores. Placas e trombose são particularmente freqüentes na artéria femoral, na altura do canal dos adutores (canal de Hunter) ${ }^{19,24}$ e na artéria poplítea na região inferior do joelho ${ }^{20,25}$. Em decorrência do comprometimento circulatório, o paciente passa a apresentar claudicação intermitente e úlceras isquêmicas nas porções mais distais do membro como no terço inferior da perna, tornozelo e dedos do pé de etiologia aparentemente espontânea, embora o trauma seja relevante. O tratamento consiste em melhorar a perfusão circulatória que se consegue mediante métodos cirúrgicos como tromboendarterectomia, "by-pass" arterial, enxerto arterial, ou métodos farmacológicos mediante bloqueios sobre o neuroeixo ou bloqueios periféricos com anestésicos locais ${ }^{21,26}$ ou com agentes neurolíticos (fenol) sobre a cadeia simpática lombar ${ }^{22,23,27,28}$. No Caso 4 , optamos por procedimentos vasodilatadores simples como os bloqueios anestésicos contínuos dos angiótomos dos territórios somáticos dos nervos ${ }^{24,29}$ comprometidos. A implantação dos catéteres venosos na altura da cabeça fibular (nervo fibular superficial) e na face medial distal da coxa (nervo safeno interno $)^{25,30}$ foram realizados pelas abordagens anatômicas e pela técnica da perda de resistência. Após confirmação radiológica (Figuras 4 e 5), os catéteres foram conectados a bombas de infusão.

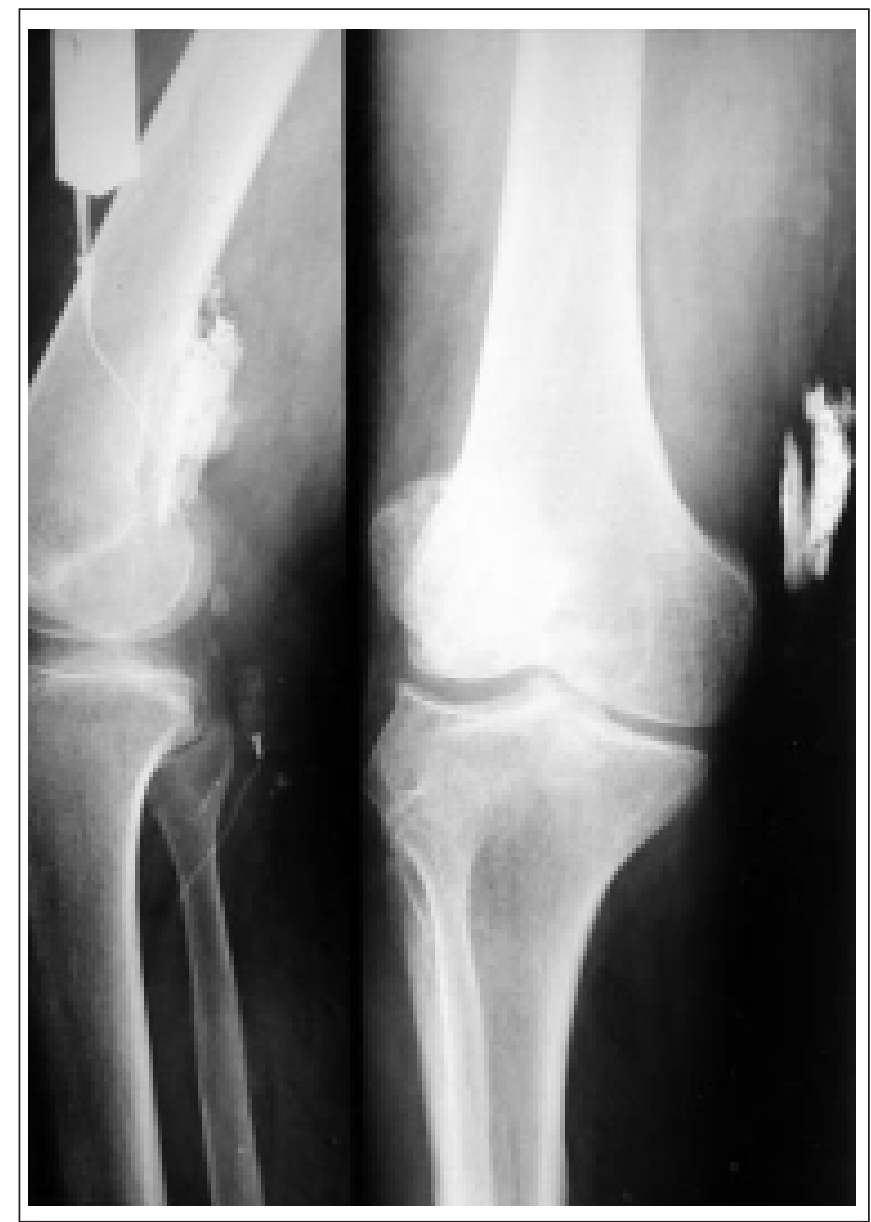

Figura 4 - Radiografias AP e Perfil, Identificando o Cateter Venoso na Altura do Nervo Safeno Interno

No Caso 5, constata-se mais um evento traumático em que a utilização de anestésicos locais apresenta uma indicação precisa. Asubstituição de analgésicos opióides por soluções anestésicas é de relevância em pediatria, pois evita os efeitos secundários dos primeiros, notadamente as manifestações de paralisia da musculatura lisa intestinal e vesical, bastante incômodas em pacientes obrigados a permanecerem no leito por tempo prolongado. Foi o que ocorreu com o paciente do Caso 5 sob terapia opióide. E mais, os marcadores de estresse cirúrgico quando se comparou a anestesia

Revista Brasileira de Anestesiologia Vol. 52, No 1, Janeiro - Fevereiro, 2002 
geral com a anestesia regional pediátrica ${ }^{26,31}$ foi favorável à última, influenciando na cicatrização, redução do período de convalescência pós-operatória. Pelo estudo radiológico associado ao exame físico do paciente, percebeu-se que a sintomatologia álgica era decorrente de dor por excesso de nocicepção e com indicação para os anestésicos locais. Para avaliar se um bloqueio anestésico do nervo femoral aliviava a dor, foi efetuado um bloqueio prova à semelhança dos $\mathrm{Ca}$ sos 2 e 3 . Uma infusão contínua com dose de reforço da solução anestésica (Figura 6) permitiu troca de curativos dolorosos e reintervenções cirúrgicas seriadas da fístula.

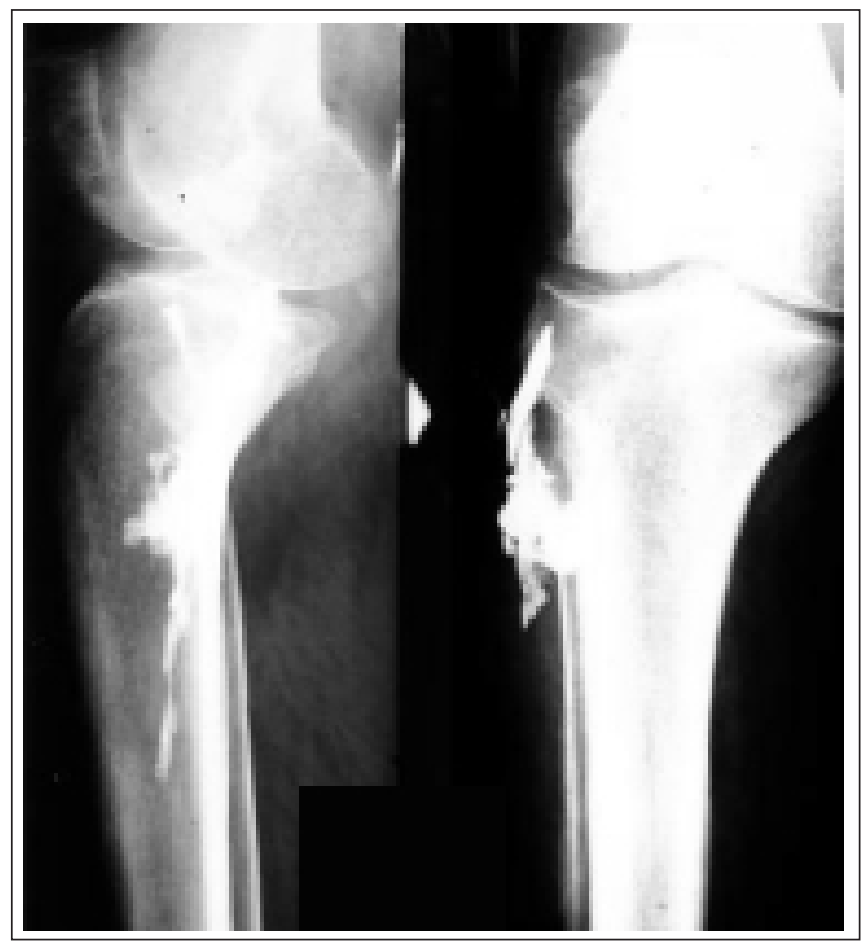

Figura 5 - Radiografias AP e Perfil Mostrando o Contraste na Cabeça da Fíbula ( Nervo Fibular)

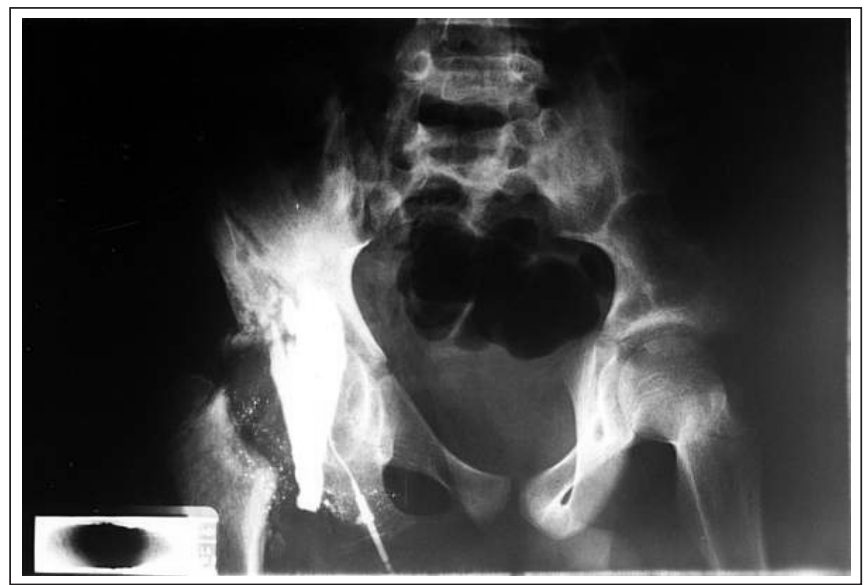

Figura 6 - Na Radiografia AP são Vistos Múltiplos Fragmentos de Chumbo de Arma de Fogo com Destruição da Cabeça do Fêmur Direito

Revista Brasileira de Anestesiologia

Vol. 52, № 1, Janeiro - Fevereiro, 2002
O nervo femoral inerva a cabeça do fêmur (esclerótomo) e a face anterior da coxa (dermátomo). Bloqueios pela abordagem paravascular femoral ${ }^{27,32}$ resultam bloqueio 2:1 (nervo femoral e nervo fêmoro-lateral cutâneo) por falhar a anestesia do nervo obturador, que pode ser bloqueado quando se utilizam: 1) catéteres longos pela mesma abordagem $28,29,33,34,2$ ) volumes maiores de anestésico, ou 3) a abordagem de Dalens ${ }^{30,35}$. Apesar da imobilização decorrente da tração transesquelética do membro inferior direito, catéteres venosos (curtos) fixados com adesivos na região inguinal são propensos a se deslocarem ao exterior. Como isso ocorreu no paciente, optamos por um cateter peridural introduzido aproximadamente dez centímetros no mesmo local através de outro cateter venoso servindo como guia. A seguir foi fixado com adesivo sob forma de "sanduíche" ${ }^{1}$, embora o método da "estrela" de Raj também seja aconselhável ${ }^{15,20}$.

Dentre as doenças autoimunes, as colagenoses agrupam o lúpus, a dermatomiosite e a esclerodermia ${ }^{31,36}$. A paciente do Caso 6 apresentava a forma de Esclerodermia Sistêmica Progressiva com manifestações vasculares de Raynaud nos quatro membros. Por comprometimento circulatório de todo membro inferior esquerdo, o pé tornou-se extremamente doloroso. Identificado o angiótomo da região ${ }^{23,28}$, processou-se a anestesia prolongada do nervo tibial posterior com doses intermitentes de bupivacaína via cateter (Figura 7). Dias depois, o processo isquêmico delimitou-se ao terceiro dedo com início de necrose que foi amputado. A permanência do cateter venoso proporcionou efetiva analgesia preemptiva, analgesia cirúrgica e analgesia pós-operatória eficiente.

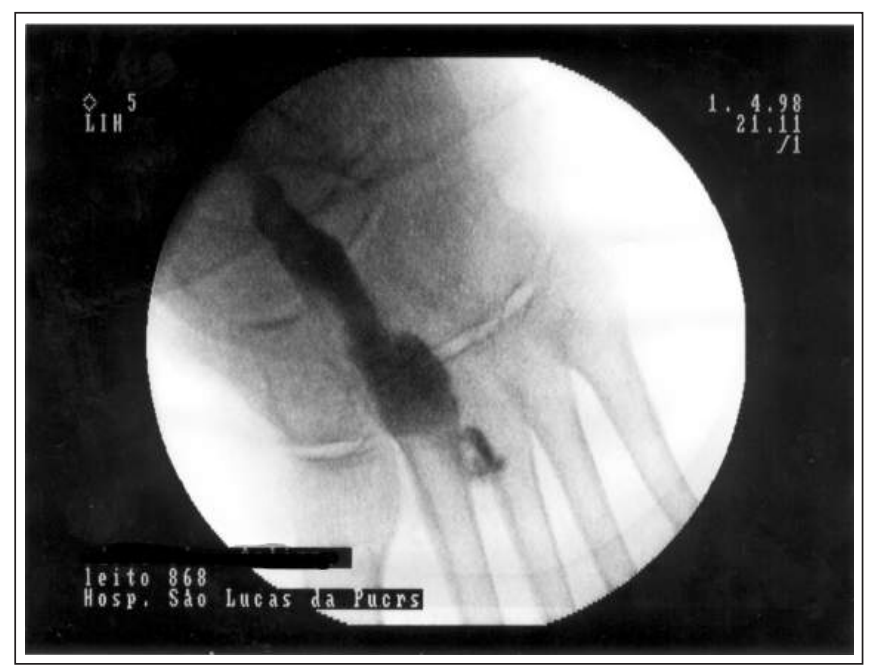

Figura 7 - Paciente Portadora de Doença Autoimune com Úlcera Isquêmica no Pé Esquerdo. Cateter Venoso foi Colocado Contíguo ao Nervo Tibial Posterior no Maléolo Medial para Analgesia do Angiótomo Correspondente

\section{CONCLUSÃO}

O conhecimento de doenças apresentadas, da anatomia tanto superficial como profunda, da farmacocinética e farmacodinâmica dos anestésicos locais, do domínio das técnicas 
anestesiológicas periféricas, a conscientização das complicações e os bons resultados demonstrados pelos catéteres curtos (venosos) e segmentos de cateter peridural permitem uma segura e boa prática de analgesia regional contínua.

\section{Prolonged Regional Analgesia with Peripheral Catheters. Case Reports}

\author{
Karl Otto Geier, M.D.
}

\section{INTRODUCTION}

Prolonged regional analgesia without systemically involving other physiological parameters resulting in hemodynamic, vascular or respiratory consequences has always been the anesthesiologist goal. Clinical situations such as: postoperative analgesia, painful dressing replacement, serial surgical reinterventions, painless physical therapy and acute and/or chronic pain sedation are common situations in hospital settings and in pain clinics. Unfortunately, due to the lack or even inexistence of compatible material and specific regional anesthesia techniques, performing most of these procedures in the above-mentioned situations was unfeasible. Alternative regional analgesia induced by repetitive punctures was uncomfortable and painful for the patient and labor-intensive for the anesthesiologist. Local long-lasting anesthetics in bolus had limited action and prolonged analgesia/anesthesia required the insertion of peripheral catheters. Basic requirements for the practice and good performance of regional anesthesia were then limited to anatomic knowledge, nervous fiber physiology, armamentarium, experience and individual skills of the anesthesiologist as to regional anesthetic techniques. However, even in the absence of optimal material, it is possible to induce blockade and continuous techniques. These reports aimed at showing that venous catheters or epidural catheter segments placed close to peripheral nerves or nervous plexus have prophylactic (phantom pain), diagnostic

(somatic pain of sympathetic pain), therapeutic (postoperative analgesia) and prognostic (evolution of sympathetic maintenance pain syndromes) objectives.

\section{CASE REPORTS}

Case 1 - Female patient, 28 years of age, operator of a shoe plant assembly line. In activating the machine, she suffered a total thumb luxation in the metacarpophalangeal region. Although reduced moments after, patient continued to refer continuous "burning-type" pain and physical inability during the following days. She was operated on aiming at "articular reinforcement". The situation worsened in the postoperative period and she was referred to the Pain Department. By the time, her hand was cold, sweaty, cyanotic and with manifestations of allodynia and hyperalgesia. The diagnosis was
Complex Regional Pain type 1. An $18 \mathrm{G}$ venous catheter was inserted in the elbow next to dermatomes and angiotomes corresponding to radial nerves and cutaneous muscle territory, aiming at sedating sympathetic activity with $8 \mathrm{ml}$ of $0.25 \%$ bupivacaine, up to four times a day. Radial nerve peripheral blockade was initially obtained by puncture with a 25 $x 7$ needle, two centimeters above the articular cutaneous fold, laterally to the biceps tendon. With the needle in such position, an analgesic test dose was injected and analgesia was observed in few minutes. Next, the needle was replaced by a venous catheter carefully reproducing previous puncture until loss of resistance between muscle planes (approximately $2 \mathrm{~cm}$ from skin). Catheter was inserted and fixed, and a radiological study confirmed its correct position (Figure 1). After each anesthetic injection, vasodilation, redness and involved territory analgesia were observed. Treatment was completed with regional intravenous blockades with local anesthetics and guanethidine. Additionally, under the effect of regional block, painless physical therapy was started. After two weeks, the symptoms were considerably better.

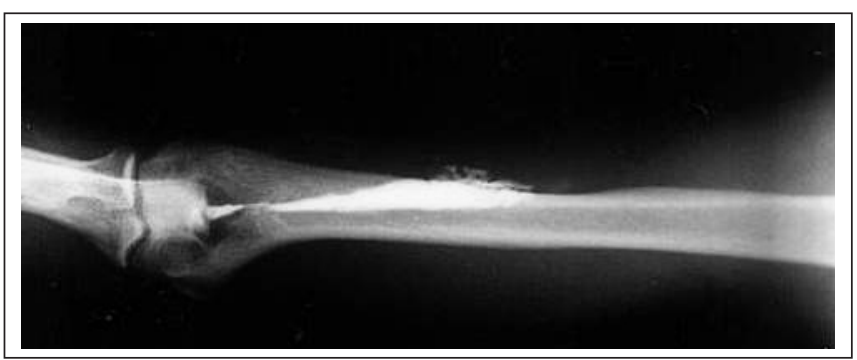

Figure 1 - Profile X-ray Identifying Venous Catheter and Contrast at the Level of Radial and Muscle-Cutaneous Nerves in Patient with Complex Regional Pain Syndrome 1

Case 2 - Male patient, 45 years of age who suffered a traffic accident and had severe rupture of the rotator cuff of right shoulder. He was operated on and needed early physical therapy in the immediate postoperative period. Unable to perform active and passive movements of the scapulohumeral joint due to pain, he was referred to the Pain Department. After thorough evaluation, an $18 \mathrm{G}$ venous catheter was inserted in the scapula supraspinal fossa, next to the suprascapular nerve, aiming at shoulder active and passive painless physical therapy. $5 \mathrm{ml}$ of $0.25 \%$ bupivacaine with epinephrine 1:200,000 20 minutes before physical therapy allowed for painless active movements for three days when the catheter was expelled. The treatment continued with NSAIDs and analgesics.

Case 3 - Female patient, 17 years of age, reached by a lost bullet which crossed the greater pectoral muscle and the left arm. Peripheral arterial beats were impaired. She was submitted to urgent revascularization of the subclavian artery with venous auto-graft. Less than 24 postoperative hours she referred hand and forearm continuous burning pain, hyperalgesia ${ }^{8}$ and allodynia ${ }^{8}$. She was initially treated with Vol. 52, Nº 1, Janeiro - Fevereiro, 2002 
analgesics and NSAIDs, however without success. Forty-eight hours after, the Pain Department was called and diagnosed left upper limb Complex Regional Pain type 2. The patient was treated for three days with a series of intermittent blockades of the left cervicothoracic sympathetic nerve through an epidural catheter segment introduced through an $18 \mathrm{G}$ venous catheter previously inserted in $\mathrm{C} 6-\mathrm{C} 7$ at the level of the stellate ganglion. Although a short-duration pain relief, analgesia was effective, especially with regards to allodynia. When the catheter was displaced to outside at the end of the third day, procedures were replaced by intermittent brachial plexus blockades for six days through a venous catheter inserted in the interscalenic cleft. Injections of $8 \mathrm{ml}$ of $0.125 \%$ bupivacaine with epinephrine $1: 400,000$ and of $30 \mathrm{ml} 0.25 \%$ bupivacaine with epinephrine 1:200,000 were used, respectively, for sympathetic activity sedation (stellate ganglion) and left upper limb somatic pain analgesia (brachial plexus) (Figure 3). Under brachial plexus analgesia, early physical therapy was started. Patient was discharged nine days later with marked improvement of symptoms.

Case 4 - Male patient, 54 years of age, with a huge painful leg ulcer of arteriosclerotic origin, located on the lower third of the leg, in territories enervated by internal saphenous and fibular D peripheral nerves, who had been submitted to a femoral-popliteal "bypass". After surgery, an infection compromised revascularization with the need for a larger iliac-popliteal bypass, however still without good results. Two free skin grafts over the ulcer resulted in approximately $80 \%$ rejection. Right lower limb remained pale, cold, painful and swollen. Without perspective of resolution, the patient was a candidate to limb amputation. After evaluation by the Pain Department, patient was submitted to $18 \mathrm{G}$ venous catheters insertion next to the referred nerves. For the fibular nerve, the catheter was inserted at the level of the head of fibula and for the internal saphenous nerve, it was inserted $8 \mathrm{~cm}$ above the femorotibial joint by the transartorial technique. After $8 \mathrm{ml}$ of $1.5 \%$ lidocaine injection in each catheter, symptoms evolved to an adequate analgesia. Continuous perfusion with low lidocaine concentrations and infusion pumps was maintained in each catheter. Six hours later the result was amazing. Left leg presented with analgesia, warm, colored and with a less ischemic ulcer. Treatment persisted for 24 hours when new grafting was performed. Catheters allowed for surgical anesthesia and postoperative analgesia. Patient was discharged the nex day and was advised to continue with the same analgesia via catheters with fractionated doses. He returned on the $7^{\text {th }}$ postoperative day for evaluation. According to the surgeon, the skin graft over the ulcer presented with $70 \%$ integration.

Case 5 - Male patient, 7 years of age, accidentally reached by a gun shot on the right flank from upwards down in medial-lateral position with low abdominal penetration and exiting at the level of the lateral thigh, with total destruction of head of femur. Patient was submitted to laparotomy and then to transkeletal traction of the right lower limb with $5 \mathrm{~kg}$. Pain at abdominal incision decreased after the third day, but he continued with severe pain in the coxofemoral joint which then presented with a purulent fistulous path through the bullet's exit hole. Patient was under opioids. After ten days, patient was asymptomatic as to the laparotomy, however femoral pain was unbearable. The Pain Department diagnosed pain due to excessive nociception (purulent drainage). Adiagnostic blockade was obtained with $5 \mathrm{ml}$ of $1.5 \%$ lidocaine in the femoral nerve. After a 4-minute onset, pain decreased until total relief after 10 minutes. The $25 \times 7$ needle that remained in place was replaced by a $20 \mathrm{G}$ venous catheter reproducing the same puncture aiming at anesthetizing the whole somatic territory supplied by the femoral nerve. Intermittent blockades with $8 \mathrm{ml}$ of $0.25 \%$ bupivacaine with epinephrine $1: 200,000$ every 6 hours during the first 24 hours and then under continuous infusion with pump resulted in surprising analgesia even during dressing replacements. Unfortunately, in the second day the catheter was expelled and had to be replaced by a new one. An epidural catheter was inserted in the same space through another venous catheter that worked as a guide, as in case 3 . With the peripheral catheter it was possible to obtain surgical anesthesia for fistula debridement and postoperative analgesia. After 15 days and still under skeletal traction, the patient was better and the fistula was almost healed.

Case 6 - Female patient, 78 years of age, with associated Systemic Lupus Erithematosus and Sclerodermia, both auto-immune diseases, with the following history: 1) Raynaud's syndrome symptoms on both hands, effectively treated with a series of anesthetic blocks on the cervicothoracic sympathetic chain (stellate ganglion); 2) several vascular surgeries on the right lower limb for the same reason. Recently, due to progressive circulatory problems typical of auto-immune diseases, she had her right lower limb amputated above the knee; 3) amputation of the second left foot toe due to an ulcer with hallux necrosis by distal circulatory failure. With ischemic pain on the third toe of the same foot also evolving to necrosis, patient was referred to the Pain Department. At evaluation, pain was unbearable. Treatment was started with a series of Bier blockades with local anesthetics, guanethidine and a microcirculation dilator. Then, a 20G venous catheter was inserted close to the posterior tibial nerve, in the internal malleolus aiming at analgesia and corresponding angiotome blockade. Atest blockade with $6 \mathrm{ml}$ of $0.25 \%$ bupivacaine resulted in total pain relief. Analgesia was maintained with infusion of the same solution every 6 hours and then under patient's request. With the necrotic process limited to the third toe, there was no need to amputate the foot as previously scheduled, but only the toe. After three days, the catheter was expelled and replaced by a new venous catheter. The second catheter remained in situ for nine days and was helpful for preemptive analgesia, the surgery itself (amputation of the third toe), and postoperative and all residual ischemic pain analgesia.

\section{DISCUSSION}

Among Complex Regional Pain Syndromes classification ${ }^{1,6}$, Complex Regional Pain 1, formerly called Reflex Sympa- 
thetic Dystrophy, corresponds to a neurological disorder caused by a nociceptive traumatic or surgical event, with clinical-pathological consequences manifested by pain and sensory abnormalities disproportional in intensity, distribution and duration ${ }^{2,7}$. The etiologic noxious effect of case 1 was trauma. In addition, motor, autonomic, trophic and psychological dysfunctions were found which resulted in regional physical inability. Burning pain is severe and extremely uncomfortable. There are allodynia ${ }^{3,8}$, hyperesthesia ${ }^{3,8}$, edema, dystrophy due to rest of the affected area, lack of vasomotor and sudomotor control (hyperhidrosis or anhidrosis) of the affected area, caused by sympathetic autonomic hyperactivity, phenomena presented by the patient. Eventually, the symptoms evolved to articular rigidity or "freezing" with severe osteoporosis confirmed by radiological exam. It is more common on extremities, hands and fingers; also shoulders, coxofemoral joint and, sometimes, post-mastectomy pectoral region. Complex Regional Pain Syndromes are worsened after recurrent noxious events in active regions and under inadequate therapy or even no therapy whatosever. Diagnosis is confirmed by history and clinical evaluation being relevant a traumatic or noxious event. In case 1, allodynia would appear after a mere blow (aeolic allodynia) on the involved areas (dermatomes). Treatment consists of trunk and regional intravenous analgesia ${ }^{4,5,9,10}$, respectively, with local anesthetics and local anesthetics/guanethidine, sympathetic hyperactivity sedation and early painless physical therapy (passive and active) under analgesia. This was our approach for the patient. Clinical history and physical evaluation associated to peripheral regional anesthetic blocks leads, very precisely, to the diagnosis ${ }^{5,10}$. The repetition of the same blockades via catheter (Figure 1) influences therapy and prognosis. The rotator cuff receives major enervation from the suprascapular nerve (C4-C6). It is a joint tendon made up of supra and infraspinous rotating muscles, small, round and subscapular, constituting the "caul" of the scapulofemoral joint ${ }^{6,11}$. Stretching injuries, traumas with complete or incomplete injury resulting in inflammation, fibrosis or cartilaginous degeneration of the glenohumeral joint are important etiologies affecting the rotator cuff. The trauma involving Case 2 patient is the major cause of hypovascularization due to constant compression involving the greater tuberosity and anterior and inferior portions of the acromium over the insertion of the supraspinous muscle and impairing its nutrition. Another major cause is the primary subacromial impact with rotator cuff degeneration, the conflict area of which is located at the insertion of the supraspinous muscle and the long biceps head. The constant attrition of those muscles against the acromial arch results in circulatory involvement and rotator cuff degeneration 7,12 . When the rupture is confirmed, surgery is in general indicated, early followed by physical therapy. Physical therapy is important because with the early movement of scapulohumeral joint, the "frozen" shoulder is prevented. Rotator cuff analgesia may be attained by interscalenic brachial plexus block, anterior ${ }^{8,13}$, posterior ${ }^{9,14}$ or intra-articular ${ }^{10,15}$ suprascapular nerve block. Depending on local anesthetics mass and concentration, the first option has the inconvenient of mildly or moderately blocking the whole superior limb, resulting in the inability for active movements or in insufficient analgesia during physical therapy. Suprascapular nerve block is the second effective alternative. In our hospital, the treatment of pain resulting from fractures isolated or not from the scapula, consists of continuous suprascapular nerve block with the patient in the sitting position, although the supine position being also described for the same disease ${ }^{11,16}$. Satisfactory results of continuous suprascapular nerve block in other situations, such as postoperative analgesia ${ }^{10,15}$ and shoulder oncologic pain ${ }^{12,17}$, have broadened such blockade indications.

Patient was approached posteriorly, with a normal $25 \times 7$ needle until supraspinal fossa paresthesia was obtained. This technique has been used for other diseases ${ }^{13,18}$, however with a modified position ${ }^{14,19}$. With the needle in place, a therapeutic test was performed with $3 \mathrm{ml}$ of $0.25 \%$ bupivacaine. After confirming analgesia, the needle was removed and puncture was reproduced with a $20 \mathrm{G}$ venous catheter introduced until the scapula was touched. First blockades were performed with patients in the sitting position and the access to the spinal fossa was vertical (Figure 2); currently, however, it is performed horizontally. With the catheter in place, the external segment is fixed with adhesive tape and protected with gauze. Analgesic $0.25 \%$ bupivacaine doses ( $5 \mathrm{ml}$ every 6 hours) provided for patient's recovery.

Duly oriented, patient and physical therapist performed, in the office and at home, painless physical therapy under bupivacaine for three days while the catheter remained in place. After seven days the patient was virtually asymptomatic. As in Case 3, Complex Regional Pain Syndromes also involve Complex Regional Pain Syndrome 2, formerly called causalgia ${ }^{1,6}$, which has a traumatic or surgical event as the underlying cause, however associated to neural injury. Most important algic manifestations are dysesthesias ${ }^{3,8}$ (acute pain in "twinges" along the injured neural tissue) and allodynia. Similarly to Complex Regional Pain Syndrome 1, diagnosis is attained by clinical history and physical evaluation. Since our intention was to separate pain type and intensity (somatic pain from sympathetic autonomic pain) ${ }^{15,20}$, we started with repetitive stellate ganglion blockades via catheter and not with successive punctures. Then, an epidural catheter segment was inserted via previously positioned venous catheter, which acted as a guide for the former catheter. Venous catheter removal was similar to the epidural space catheterization technique. A prolonged stellate ganglion sympathetic activity sedation had been obtained in previous situations ${ }^{16,17,21,22}$. With low intermittent or continuous local anesthetic concentrations under PCA ${ }^{17,22}$, sympathetic maintenance pain becomes more controlled. However, the short stay of the catheters in situ, probably due to unfavorable anatomic region, constant cervical movement and catheter fixation with adhesive tape made unfeasible a prolonged treatment. For this reason, we continued with the interscalenic treatment (Figure 3 ) with an $18 \mathrm{G}$ venous catheter inserted differently from the approach (needle direction) 
and the technique (loss of resistance) preconized by Winnie ${ }^{18,23}$, which is a routine in our hospital. Patient was discharged after nine days and continued with outpatient therapeutic treatment.

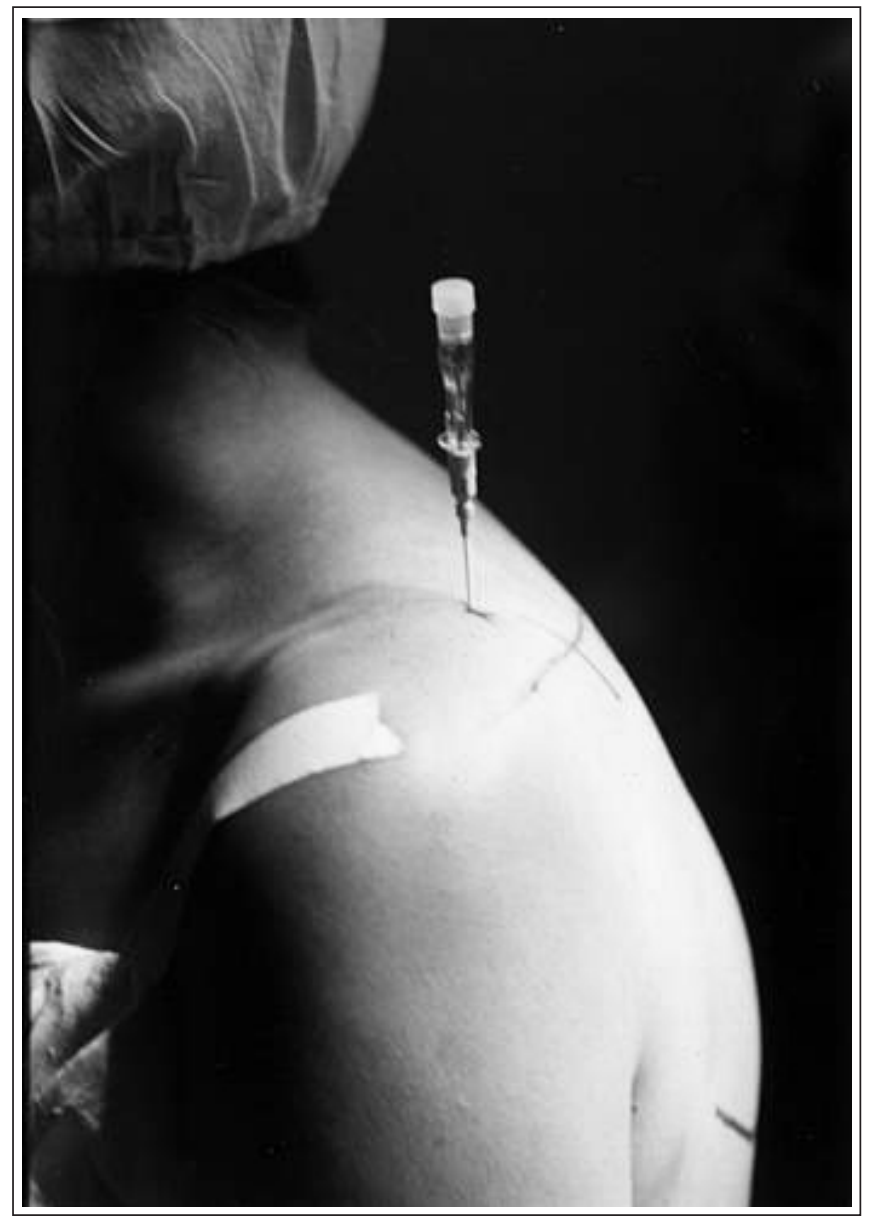

Figure 2 - Venous Catheter in the Supraspinal Space of Left Scapula

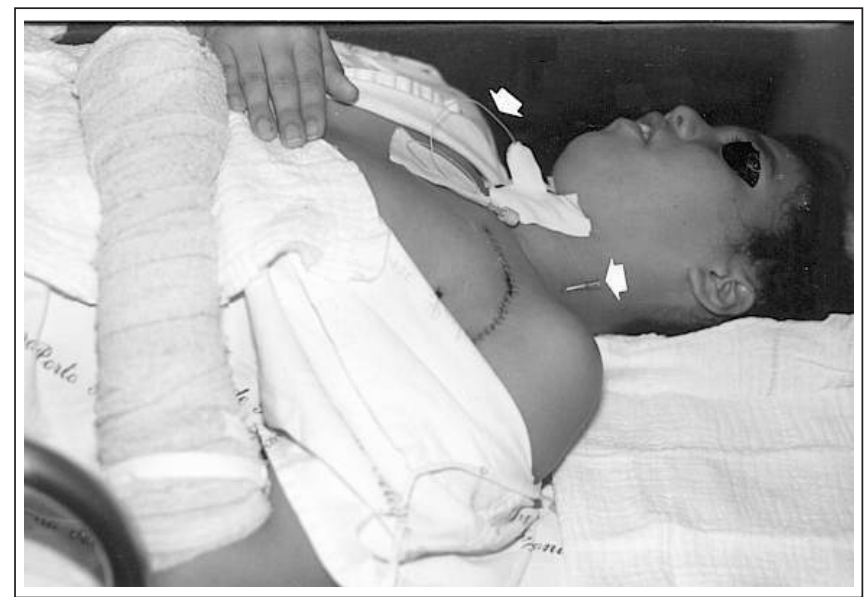

Figure 3 - Patient with Complex Regional Pain Syndrome 2 by firearm shot which crossed greater pectoral muscle with subclavian and brachial plexus vascular injury. Intermittent stellate ganglion blockade via epidural catheter segment (arrow) was effective while in situ. Therapy continued via interscalenic venous catheter (arrow)

Revista Brasileira de Anestesiologia

Vol. 52, № 1, Janeiro - Fevereiro, 2002
Obliterating arteriosclerosis is the most common arterial disease of the inferior abdominal aorta, the iliac artery and its lower limb branches. Plates and thrombosis are particularly frequent in the femoral artery at the adductors channel level (Hunter channel) ${ }^{19,24}$ and in the popliteal artery in the lower knee ${ }^{20,25}$. As a consequence of circulatory involvement, patients present intermittent claudication and ischemic ulcers in the most distal parts of the limb, such as lower third of the leg, ankles and toes, with a seemingly spontaneous etiology, although trauma being relevant. Treatment consists in improving circulatory perfusion by surgical methods such as thromboendarterectomy, arterial bypass, arterial graft, by pharmacological methods by blocking the neuraxis or by peripheral blocks with local anesthetics ${ }^{21,26}$ or neurolitic agents (phenol) on the lumbar sympathetic chain ${ }^{22,23,27,28}$. In Case 4 , we decided for simple vasodilating procedures, such as continuous anesthetic blocks of the angiotomes of involved nerves somatic territories ${ }^{24,29}$. Venous catheter insertion at the fibular head level (superficial fibular nerve) and in the distal medial face of the thigh (internal saphenous nerve) ${ }^{25,30}$ were performed by anatomical approaches and by the loss of resistance technique. After radiological confirmation (Figures 4 and 5), catheters were connected to infusion pumps.

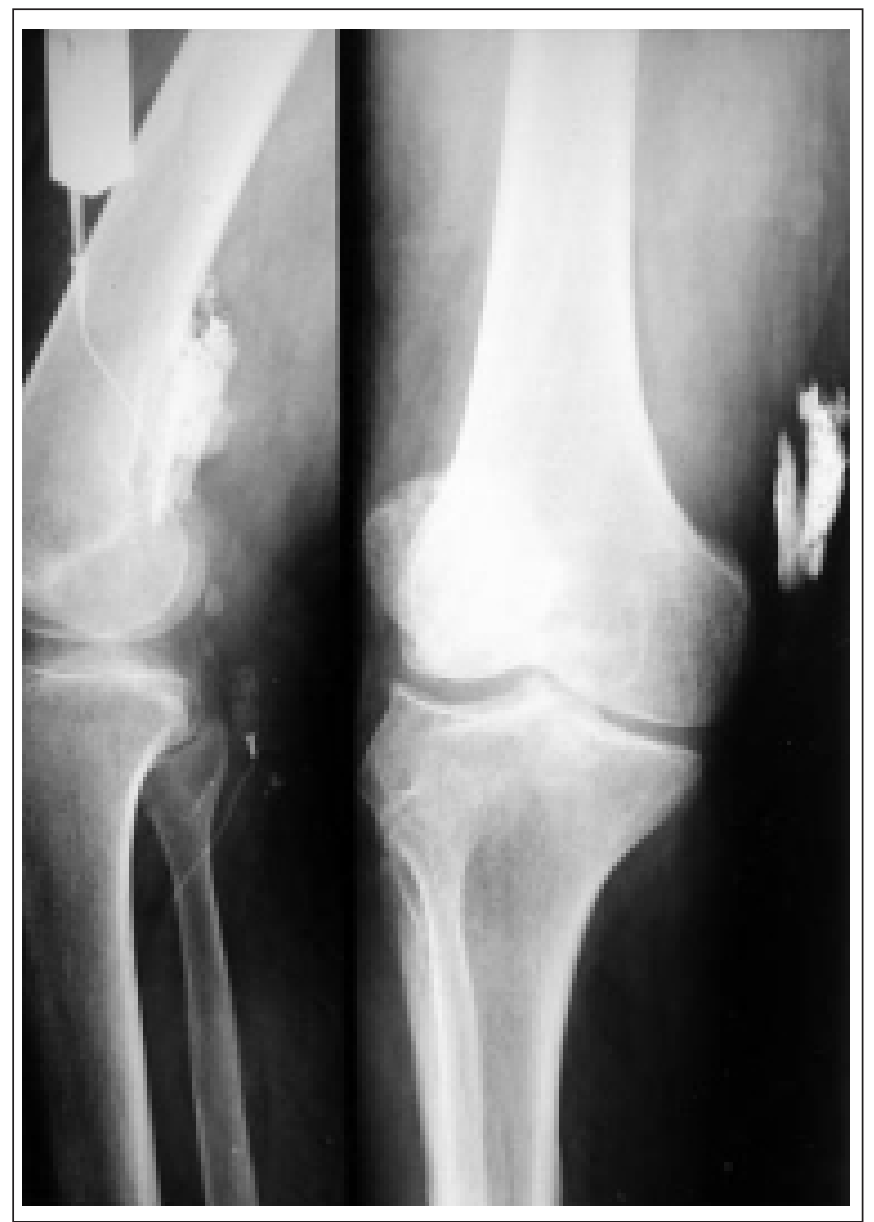

Figure 4 - PA and Profile X-Rays, Identifying Venous Catheter at the Level of Internal Saphenous Nerve 


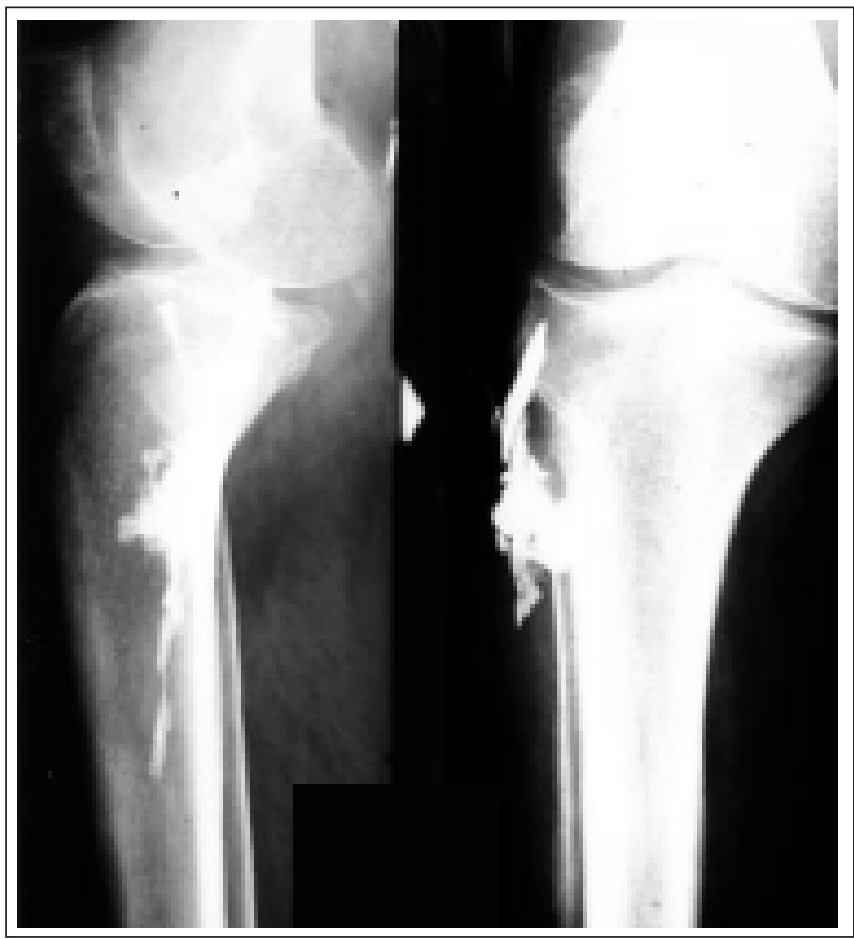

Figure 5 - PA and Profile X-Rays Showing Head of Fibula Contrast

Case 5 presented with a traumatic event where the use of local anesthetics has a precise indication. The replacement of opioids by anesthetic solutions is relevant in pediatrics because it prevents secondary effects of opioids, especially intestinal and vesical smooth muscles palsy, very uncomfortable for patients forced to stay in bed for long periods. This was the situation in Case 5, under opioid therapy. Moreover, surgical stress markers, when general anesthesia is compared to regional pediatric anesthesia ${ }^{26,31}$, were favorable to the latter, positively influencing healing and postoperative recovery. The radiological exam associated to physical evaluation has shown that pain was caused by excessive nociception with indication for local anesthetics. To evaluate whether the femoral nerve anesthetic block would relieve pain, a test block was induced, similarly to Cases 2 and 3 . Continuous infusion with an anesthetic booster (Figure 6) allowed for the replacement of painful dressings and serial surgical reinterventions in the fistula.

The femoral nerve enervates the head of femur (sclerotome) and the anterior face of the thigh (dermatome). Paravascular femoral blocks ${ }^{27,32}$ resulted in blockade 2:1 (femoral nerve and cutaneous femorolateral nerve) due to failure of obturator nerve anesthesia, which may be obtained when using: 1) long catheters and the same approach $28,29,33,34,2$ ) higher anesthetic volumes, or 3) Dalens' approach ${ }^{30,35}$. In spite of the immobilization provided by the transkeletal traction of the right lower limb, venous catheters (short) fixed with adhesives on the inguinal region tend to displace to outside. Since this was the case with the patient, we decided for an epidural catheter inserted approximately ten centimeters from the same site through another venous catheter acting as a guide. Then, it was fixed with adhesive as a "sandwich" ${ }^{1}$, although Raj's "star" method is also recommended ${ }^{15,20}$.

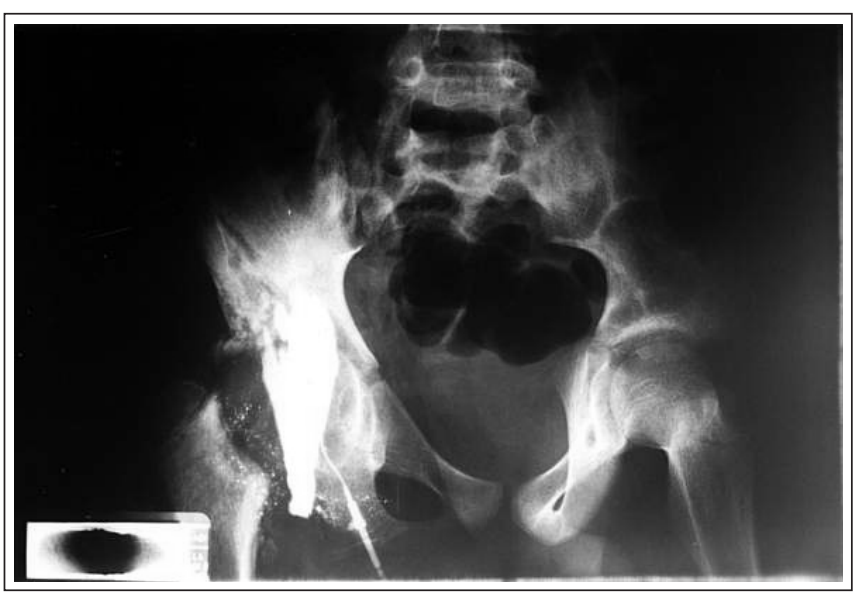

Figure 6 - PA X-Ray showing Multiple Lead Fragments of Firearm with Head of Right Femur Destruction

Lupus, dermatomyositis and scleroderma ${ }^{31,36}$ are autoimmune diseases. Case 6 patient presented with Progressive Systemic Scleroderma with Raynaud's vascular manifestations on the four limbs. Due to total circulatory involvement of the left lower limb, the foot became extremely painful. After identifying the adequate angiotome ${ }^{23,28}$, prolonged anesthesia of the posterior tibial nerve with intermittent bupivacaine doses via catheter was induced (Figure 7). Some days later, ischemia with early necrosis was restricted to the third toe, which was amputated. The venous catheter has provided effective preemptive, surgical and postoperative analgesia.

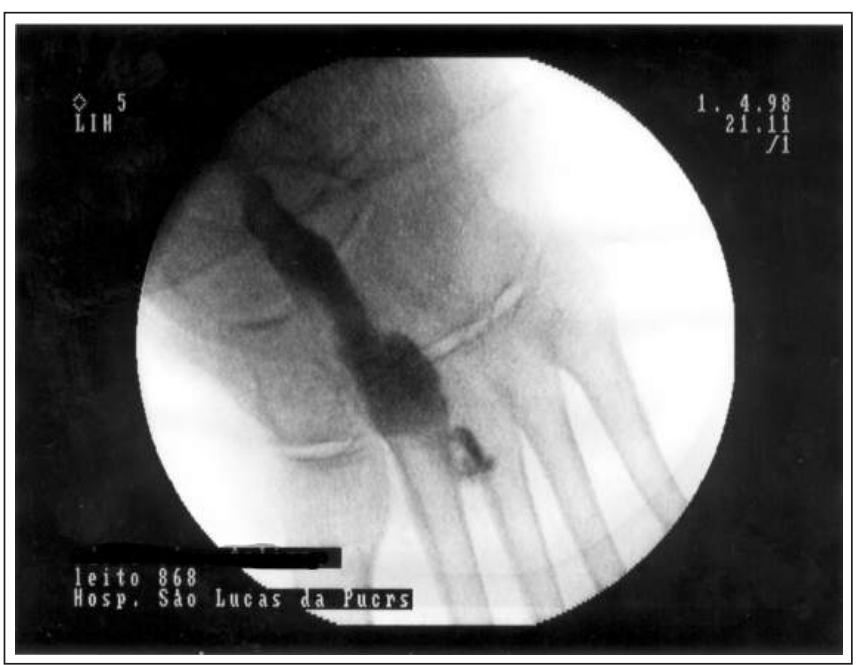

Figure 7 - Autoimmune Disease Patient with Left Foot Ischemic Ulcer. Venous Catheter was Placed Next to the Posterior Tibial Nerve in the Medial Malleolus for Corresponding Angiotome Analgesia 


\section{CONCLUSIONS}

The knowledge of the diseases, of both superficial and deep anatomy, of local anesthetics pharmacokinetics and pharmacodynamics and of peripheral anesthetic techniques; the awareness of the complications and the good results shown by short (venous) catheters and epidural catheter segments allow for a safe and good continuous regional anesthesia.

\section{REFERÊNCIAS - REFERENCES}

01. Dunn DG - Chronic Regional Pain Syndrome, Type 1; Part 1. AORN J, 2000;72:421-449.

02. Bonica JJ - Definitions and Taxonomy of Pain, em: Bonica JJ The Management of Pain. $2^{\text {nd }}$ Ed, Philadelphia, Lea\&Febiger, 1990;18-27.

03. Muller A, Sudbrack G - Sympatholyse par voie veineuse dans les artériopathies oblitérantes et dans les acrosyndromes. Agressologie 1990;31:203-206.

04. Boas RA, Cousins MJ - Diagnostic Neural Blockade, em: Cousins RA, Bridenbaugh PO - Neural Blockade. $2^{\text {nd }} \mathrm{Ed}$, Lippincott. Philadelphia, 1998;885-898.

05. Cailliet R - L'Épaule.Masson. Paris, $10^{\mathrm{a}}$ Ed, 1985;1-34.

06. Lech O - Fundamentos em Cirurgia do Ombro. São Paulo. Editora Harbar, 1995;75-96

07 . Wassef MR - Suprascapular nerve block. Anaesthesia, 1992;47:120-124.

08. Moore DC - Block of the Suprascapular Nerve. Illinois. Charles C Thomas Publisher, 1953;225-228.

09. Rawal N, Hylander J, Allvin R et al - Patient-Controlled Regional Analgesia (PCRA) After Day Surgery. The International Monitor. Medicon International $16^{\text {th }}$ Annual ESRA Congress. London, 1997;9:3:179.

10. Breen TW, Haigh JD - Continuous suprascapular nerve block for analgesia of scapular fracture. Can J Anaesth, 1990;37: 786-788.

11. Mercadante S, Sapio M, Villari P - Suprascapular nerve block by catheter for breakthrough shoulder cancer pain. Reg Anesth, 1995;20:343-346.

12. Geier KO - Bloqueo del Nervio Supraescapular (BNSS) para Manejo del Dolor en la Articulación Escapulo Umeral. IV Congreso Panamericano de Anestesia Regional, 1998;129.

13. Parris WC - Suprascapular nerve block: a safer technique. Anesthesiology, 1990;72:580-581.

14. Raj PP - Prognostic and Therapeutic Local Anesthetic Blockade, em: Cousins MJ, Bridenbaugh PO - Neural Blockade. $2^{\text {nd }} E d$, Lippincott. Philadelphia, 1998;899-933.

15. Owen-Falkenberg A, Olsen KS - Continuous stellate ganglion blockade for reflex sympathetic dystrophy. Anesth Analg, 1992;75:1041-1042.

16. Elias M, Chakerian MU - Repeated stellate ganglion blockade using a catheter for pediatric herpes zoster ophthalmicus. Anesthesiology, 1994;80:950-952.

17. Winnie AP - Interscalene brachial plexus block. Anesth Analg Cur Res, 1970;49:455-466.

18. Netter FH - Atlas of Human Anatomy. Novartis. New Jersey. Ninth printing, 1997;470.

19. Bonica JJ - Pain Due Vascular Disease, em: Bonica JJ - The Management of Pain. $2^{\text {nd }}$ Ed, Philadelphia, Lea\&Febiger. 1990;502-537
20. Bonica JJ, Buckley FP - Regional Anesthesia with Local Anesthetics, em: Bonica JJ - The Management of Pain. $2^{\text {nd }} E d$, Philadelphia, Lea\&Febiger. 1990;1883-1966

21. Bonica JJ, Buckley FP, Murphy TM - Neurolytic Blockade and Hypophysectomy, em, Bonica JJ - The Management of Pain. $2^{\text {nd }}$ Ed, Philadelphia, Lea\&Febiger. 1990;1980-2043

22. Stanton Hicks M - Lumbar Sympathetic Nerve Block and Neurolysis, em: Waldman SD, Winnie AP - Interventional Pain Management. Philadelphia. WB Saunders Company, 1996; 353-359

23. Bonica JJ - Sympathetic And Somatic Segmental And Peripheral Nerve Supply To The Hips And Lower Limbs, em, Bonica JJ - The Management of Pain. $2^{\text {nd }} E d$, Philadelphia, Lea\&Febiger, 1990;1421-1423

24. van der Wal M, Lang SA, Yip RW - Transsartorial approach for saphenous nerve block. Can J Anaesth, 1993;40:542-546.

25. Ivani G - Modification of the Stress Response by Regional Analgesia in Children, em: Van Zundert - Highlights in Pain Therapy and Regional Anaesthesia. VI. XVI Annual ESRA Congress. London. Permanyer Publications. 1997;17-20:199-202

26. Winnie AP, Ramamurthy S, Durrani Z - The inguinal paravascular technique of lumbar plexus anesthesia: the "3:1 block". Anesth Analg, 1973;52:989-996.

27. Singelyn FJ, Contreras V, Gouverneur JM - Epidural anesthesia complicating continuous 3-in-1 lumbar plexus blockade. Anesthesiology, 1995;83:217-220

28. Geier KO - Sobre o bloqueio "3:1". Rev Bras Anestesiol, 2001; 51:176-182

29. Dalens B, Vanneuville G, Tanguy A - Comparison of the fascia iliaca compartment block with the 3-in-1 block in children. Anesth Analg, 1989;69:705-713

30. Rassner G - Dermatologia. São Paulo, Livraria Editora Santos.

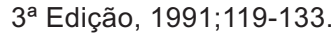

\section{RESUMEN}

Geier KO - Analgesia Regional Prolongada con Catéteres Periféricos. Relato de Casos

Justificativa y Objetivos - Síndromes de Dolor de Manutención Simpática, Tromboangiitis Obliterante, Esclerodermia Sistémica Progresiva (enfermedad auto-inmune) y analgesia pós-operatoria responden muy bien a los anestésicos locales cuando en uso prolongado El objetivo de los casos relatados tiene por finalidad divulgar el uso de catéteres cortos (catéteres venosos) el segmento de catéter peridural en las situaciones arriba nombradas, en la falta de material apropiado (Contiplex ${ }^{\circledR}$ y similares) asociados al estimulador de nervio periférico.

Relato de los Casos - Son relatados casos de Síndrome de Dolor Regional Complejo 1 y 2 , úlcera de pierna por tromboangiitis obliterante para preparación de enjerto de piel, esclerodermia sistémica progresiva con comprometimiento microcirculatorio distal del pie, en que fueron colocados catéteres venosos contiguos a los nervios periféricos de los respectivos territorios, teniendo por finalidad el control continuo del dolor a través de inyecciones de anestésicos locales.

Conclusiones - Apoyados en los principios que nortean la buena práctica de anestesia regional y en los resultados obtenidos, los casos presentados mostraron que los catéteres usados pueden substituir aquellos especialmente designados para las mismas finalidades. 